\title{
A Dirichlet problem on the half-line for nonlinear equations with indefinite weight
}

\author{
Zuzana Došlá ${ }^{1}$ • Mauro Marini² ${ }^{2}$ Serena Matucci $^{2}$
}

Received: 20 April 2015 / Accepted: 18 February 2016 / Published online: 5 March 2016

(C) Fondazione Annali di Matematica Pura ed Applicata and Springer-Verlag Berlin Heidelberg 2016

\begin{abstract}
We study the existence of positive solutions on the half-line $[0, \infty)$ for the nonlinear second-order differential equation

$$
\left(a(t) x^{\prime}\right)^{\prime}+b(t) F(x)=0, \quad t \geq 0,
$$

satisfying Dirichlet-type conditions, say $x(0)=0, \lim _{t \rightarrow \infty} x(t)=0$. The function $b$ is allowed to change sign, and the nonlinearity $F$ is assumed to be asymptotically linear in a neighborhood of zero and infinity. Our results cover also the cases in which $b$ is a periodic function for large $t$ or it is unbounded from below.
\end{abstract}

Keywords Second-order nonlinear differential equation - Boundary value problem on the half-line · Dirichlet conditions · Globally positive solution · Disconjugacy · Principal solution

Mathematics Subject Classification Primary 34B40 · Secondary 34B18

Zuzana Došlá

dosla@math.muni.cz

Mauro Marini

mauro.marini@unifi.it

Serena Matucci

serena.matucci@unifi.it

1 Department of Mathematics and Statistics, Masaryk University, Kotlářská 2, 61137 Brno, Czech Republic

2 Department of Mathematics and Informatics "Ulisse Dini”, University of Florence, 50139 Florence, Italy 


\section{Introduction}

Consider the boundary value problem (BVP) on the half-line $[0, \infty)$

$$
\begin{aligned}
& \left(a(t) x^{\prime}\right)^{\prime}+b(t) F(x)=0, \\
& x(0)=0, \quad x(t)>0 \text { on }(0, \infty), \quad \lim _{t \rightarrow \infty} x(t)=0,
\end{aligned}
$$

where we assume the following:

(i) The function $a$ is continuous on $[0, \infty), a(t)>0$, and

$$
\int_{0}^{\infty} \frac{1}{a(t)} \mathrm{d} t<\infty .
$$

(ii) The function $b$ is continuous on $[0, \infty)$, nonnegative and not identically zero on $[0,1]$, and is allowed to change sign for $t>1$. Moreover, $b$ is bounded from above, that is, there exists a positive constant $B$ such that

$$
b(t) \leq B \text { on }[1, \infty)
$$

(iii) The function $F$ is continuous on $\mathbb{R}, F(u) u>0$ for $u \neq 0, F$ is differentiable on $[0, \infty)$ with bounded nonnegative derivative:

$$
0 \leq \frac{d F(u)}{d u} \leq 1 \text { for } u \geq 0,
$$

and satisfies

$$
\lim _{u \rightarrow 0^{+}} \frac{F(u)}{u}=k_{0}, \quad \lim _{u \rightarrow \infty} \frac{F(u)}{u}=k_{\infty}
$$

where

$$
0 \leq k_{0} \neq k_{\infty}
$$

Observe that (5) implies that $k_{0}, k_{\infty} \leq 1$.

The BVP (1)-(2) is a Dirichlet-type BVP on an unbounded domain. Recently, there has been a growing interest in studying infinite interval problems associated with second-order nonlinear differential equations, under various points of view. For a wide bibliography, we refer the reader to $[1,2,22]$ and the references therein. When the weight $b$ is of fixed sign or it is sign-indefinite, we refer to $[7,15,27]$ or $[16,17,24,25]$, respectively. The BVP (1)(2) arises in the investigation of positive radial solutions for elliptic equations, when the nonlinearity is asymptotically linear, see, e.g., [3].

Our main aim is to continue this study when the function $b$ is allowed to change its sign and the nonlinearity $F$ can be, roughly speaking, close to a linear function. The investigated problem can be viewed as an extension to the half-line of recent results on nonlinear BVPs with a sign-indefinite weight on a compact interval, see, e.g., [4,5], and reference therein for a brief survey on this topic.

Denote by $|\cdot|_{L}$ the norm in $L^{1}[0,1]$ and set

$$
A(t)=\int_{0}^{t} \frac{1}{a(s)} \mathrm{d} s .
$$

Our main result is the following, in which the disconjugacy of a suitable auxiliary differential linear equation plays a key role, see Sect. 3 below. 
Theorem 1 Assume that the linear differential equation

$$
v^{\prime \prime}+\frac{B}{a(t)} v=0
$$

is disconjugate on $[1, \infty)$, where the constant $B$ is defined in (4).

If there exist $t_{1}, t_{2} \in(0,1), t_{1}<t_{2}$ such that $\int_{t_{1}}^{t_{2}} b(t) d t>0$, and

$$
\begin{aligned}
& 0 \leq \min \left\{k_{0}, k_{\infty}\right\} A(1) \quad|b|_{L}<1, \\
& \max \left\{k_{0}, k_{\infty}\right\} \int_{t_{1}}^{t_{2}} b(t) d t>\frac{A(1)}{A\left(t_{1}\right)\left(A(1)-A\left(t_{2}\right)\right)},
\end{aligned}
$$

then the BVP (1)-(2) has a solution.

Moreover, the solution $x$ has a local maximum in the interval $(0,1]$, is decreasing in $[1, \infty)$ and satisfies

$$
\int_{1}^{\infty} \frac{1}{a(t) x^{2}(t)} d t=\infty
$$

Theorem 1 covers also the cases in which the weight $b$ is a periodic function for large $t$ or it is unbounded from below.

Our approach is based on a shooting method and a continuity result. More precisely, Theorem 1 is proved by considering two auxiliary BVPs, the first one on the compact interval $[0,1]$, where $b$ is nonnegative, and the second one on the half-line $[1, \infty)$, where $b$ is allowed to change its sign. The problem of the existence of solutions for (1), emanating from zero, positive in the interval $(0,1)$, and satisfying additional assumptions at $t=1$, is considered in Sect. 2 and is solved by using some results from [23], with minor changes. The BVP on $[1, \infty)$ is examined in Sect. 4. It deals with positive decreasing solutions on $[1, \infty)$ for (1) which tend to zero as $t \rightarrow \infty$. This second problem is solved by using a fixed point theorem for operators defined in a Fréchet space by a Schauder's linearization device, see [12, Theorem 1.3]. This method does not require the explicit form of the fixed point operator, but only some a-priori bounds. These estimations are obtained using some properties of principal solutions of disconjugate second-order linear equations, see [21, Chapter 11]. Finally, roughly speaking, the solvability of (1)-(2) is obtained by using a shooting method on $[0,1]$ and, by means some continuity arguments, pasting a solution of (1) on $[0,1]$ with a solution of the BVP on $[1, \infty)$. This last argument can be viewed as a generalization to noncompact intervals of some ideas in [20].

Notice that our approach allows us to obtain also an estimation of the decay to zero of solutions of (1)-(2). Some examples complete the paper.

\section{Two auxiliary BVPs on $[0,1]$}

In this section, we recall some results about the existence of solutions of (1) on [0, 1], which belong either to $\Delta_{1}$ or to $\Delta_{2}$, where

$$
\begin{aligned}
& \Delta_{1}=\{u \in C[0,1]: u(0)=u(1)=0, u(t)>0 \text { on }(0,1)\} \\
& \Delta_{2}=\left\{u \in C[0,1]: u(0)=u^{\prime}(1)=0, u(t)>0 \text { on }(0,1]\right\} .
\end{aligned}
$$

These results can be obtained from [23], with minor changes.

BVPs on a compact interval, associated with equations of the form

$$
z^{\prime \prime}+g(t) F(z)=0
$$


where $g$ is a continuous nonnegative function on $[0,1]$, have been widely investigated in the literature, under many different points of view. We refer to [6, Introduction] and references therein for a brief survey.

In particular, the existence of solutions of (12), which satisfy either $z \in \Delta_{1}$ or $z \in \Delta_{2}$, has been considered in [19], where the key conditions on the nonlinearity are either that $F$ is superlinear, that is, $k_{0}=0, k_{\infty}=\infty$, or that $F$ is sublinear, that is, $k_{0}=\infty, k_{\infty}=0$. When the nonlinearity $F$ is not necessarily superlinear nor sublinear, these results have been extended in several ways in [23].

Using [23, Corollaries 3.1 and 3.5] and the continuity of $g$, we obtain the following result.

Lemma 1 Assume that there exist $t_{1}, t_{2} \in(0,1), t_{1}<t_{2}$, such that $\int_{t_{1}}^{t_{2}} g(t) d t>0$ and

$$
0 \leq \min \left\{k_{0}, k_{\infty}\right\}|g|_{L}<1, \quad \max \left\{k_{0}, k_{\infty}\right\} \int_{t_{1}}^{t_{2}} g(t) d t>\frac{1}{t_{1}\left(1-t_{2}\right)} .
$$

Then (12) has both solutions $z_{1} \in \Delta_{1}$ and $z_{2} \in \Delta_{2}$.

Proof In virtue of the continuity of $g$, every nonnegative solution $z$ of $(12), z \not \equiv 0$, satisfies $z(t)>0$ on $(0,1)$, since $z^{\prime}$ is nonincreasing. Hence, the assertion follows from [23, Corollaries 3.1 and 3.5].

When $g$ does not have zeros on $[0,1]$, from Lemma 1 we obtain the following.

Lemma 2 Let $g$ be positive on $[0,1]$. If

$$
0 \leq \min \left\{k_{0}, k_{\infty}\right\}|g|_{L}<1, \quad \max \left\{k_{0}, k_{\infty}\right\} \min _{t \in[0,1]} g(t)>27,
$$

then (12) has both solutions $z_{1} \in \Delta_{1}$ and $z_{2} \in \Delta_{2}$.

Proof Fixed $t_{1}, t_{2} \in(0,1), t_{1}<t_{2}$, we have

$$
\int_{t_{1}}^{t_{2}} g(\tau) \mathrm{d} \tau \geq\left(t_{2}-t_{1}\right) \min _{t \in[0,1]} g(t)
$$

Thus, the second condition in (13) is satisfied if

$$
\max \left\{k_{0}, k_{\infty}\right\} \min _{t \in[0,1]} g(t) \geq \frac{1}{t_{1}\left(1-t_{2}\right)\left(t_{2}-t_{1}\right)}
$$

for a suitable choice of $t_{1}, t_{2}$. Put $\rho=\varrho\left(t_{1}, t_{2}\right)=t_{1}\left(1-t_{2}\right)\left(t_{2}-t_{1}\right)$, it is easily checked that $\varrho$ takes its maximum $1 / 27$ on the region $0 \leq t_{1}<t_{2} \leq 1$ when $t_{1}=1 / 3, t_{2}=2 / 3$. Therefore, the second inequality in (14) follows.

Define for $t \in[0,1]$

$$
\tau(t)=\frac{A(t)}{A(1)},
$$

where $A$ is given in (7). Thus, $\tau$ maps the interval $[0,1]$ into itself. Let $x$ be a solution of (1) on $[0,1]$ and put $z(\tau)=x(t(\tau))$, where $t(\tau)$ is the inverse function of $\tau(t)$. Then, $z$ is a solution on $[0,1]$ of

$$
\frac{\mathrm{d}^{2} z}{\mathrm{~d} \tau^{2}}+\tilde{b}(\tau) F(z)=0,
$$

where $\tilde{b}(\tau)=A^{2}(1) a(t(\tau)) b(t(\tau))$. Vice versa, if $z$ is a solution of (16) on [0,1], then $x(t)=z(\tau(t))$ is a solution of (1) on the same interval. Moreover, it is easy to show that $x$ belongs to $\Delta_{i}$ if and only if $z \in \Delta_{i}, i=1,2$. Hence, Lemmas 1 and 2 read for (1) as follows. 
Proposition 1 Assume that one of the following conditions is satisfied.

(i) There exist $t_{1}, t_{2} \in(0,1), t_{1}<t_{2}$ such that $\int_{t_{1}}^{t_{2}} b(t) d t>0$, and

$$
\begin{aligned}
& 0 \leq \min \left\{k_{0}, k_{\infty}\right\} A(1)|b|_{L}<1, \\
& \max \left\{k_{0}, k_{\infty}\right\} \int_{t_{1}}^{t_{2}} b(t) d t>\frac{A(1)}{A\left(t_{1}\right)\left(A(1)-A\left(t_{2}\right)\right)} .
\end{aligned}
$$

(ii) $b(t)>0$ on $[0,1]$ and

$$
\begin{aligned}
& 0 \leq \min \left\{k_{0}, k_{\infty}\right\} A(1)|b|_{L}<1, \\
& 27<\max \left\{k_{0}, k_{\infty}\right\} A(1) \min _{t \in[0,1]} b(t) .
\end{aligned}
$$

Then (1) has both solutions $x_{1} \in \Delta_{1}$ and $x_{2} \in \Delta_{2}$.

Proof Since

$$
\int_{0}^{1} \tilde{b}(\tau) \mathrm{d} \tau=A^{2}(1) \int_{0}^{1} b(t(\tau)) a(t(\tau)) \mathrm{d} \tau=A(1) \int_{0}^{1} b(t) \mathrm{d} t=A(1)|b|_{L}
$$

and

$$
\int_{t_{1}}^{t_{2}} b(t) \mathrm{d} t=\frac{1}{A(1)} \int_{\tau_{1}}^{\tau_{2}} \tilde{b}(\tau) \mathrm{d} \tau
$$

where $\tau_{i}=\tau\left(t_{i}\right)=A\left(t_{i}\right) / A(1), i=1,2$, the assertion follows from Lemmas 1 and 2 .

Other sufficient conditions for the existence of solutions of (1) in the sets $\Delta_{1}$ and $\Delta_{2}$ can be obtained in a similar way from other results in [23].

\section{Principal solutions and disconjugacy}

Consider the linear equation

$$
\left(a(t) y^{\prime}\right)^{\prime}+\beta(t) y=0,
$$

where $\beta$ is a continuous function for $t \geq T \geq 0$. In our study, an important role is played by the disconjugacy property and the notion of principal solutions for (17).

We recall that (17) is said to be disconjugate on an interval $I \subset[T, \infty)$ if any nontrivial solution of (17) has at most one zero on $I$. We refer to [14,21] and references therein for basic properties of disconjugacy. In particular, the following results will be useful in the sequel.

Lemma 3 Let $T_{1} \geq T$. The following statements are equivalent.

$\left(\mathrm{i}_{1}\right)$ Equation (17) is disconjugate on $\left[T_{1}, \infty\right)$;

(i2) Equation (17) is disconjugate on $\left(T_{1}, \infty\right)$;

(i $\left.\mathrm{i}_{3}\right)$ Equation (17) has a solution without zeros on $\left(T_{1}, \infty\right)$.

Proof $\left(\mathrm{i}_{1}\right) \Longleftrightarrow\left(\mathrm{i}_{2}\right)$. If (17) is disconjugate on $\left[T_{1}, \infty\right)$, then it is disconjugate on $\left(T_{1}, \infty\right)$. The vice versa follows from [14, Theorem 2,Chapt.1], with minor changes. Finally, $\left(\mathrm{i}_{2}\right) \Longleftrightarrow\left(\mathrm{i}_{3}\right)$ follows from [21, Corollary 6.1].

The concept of principal solution was introduced in 1936 by W. Leighton and M. Morse and, later on, analyzed by P. Hartman and A. Wintner, see, e.g., [21, Chapter 11]. If (17) is nonoscillatory, then there exists a solution $u_{0}$ of (17), which is uniquely determined up to a 
constant factor by one of the following conditions (in which $u$ denotes an arbitrary solution of (17), linearly independent of $u_{0}$ ):

$$
\begin{aligned}
& \lim _{t \rightarrow \infty} \frac{u_{0}(t)}{u(t)}=0, \\
& \frac{u_{0}^{\prime}(t)}{u_{0}(t)}<\frac{u^{\prime}(t)}{u(t)} \quad \text { for large } t \\
& \int_{t_{u}}^{\infty} \frac{d t}{a(t) u_{0}^{2}(t)}=\infty
\end{aligned}
$$

where $t_{u} \geq T$ is such that $u_{0}(t) \neq 0$ on $\left[t_{u}, \infty\right)$. The solution $u_{0}$ is called principal solution of (17), and any solution $u$ of (17), which is linearly independent of $u_{0}$, is called a nonprincipal solution of (17). Property (18) is the simplest and most typical property characterizing principal solutions, because, roughly speaking, it means that the principal solution is the smallest one in a neighborhood of infinity.

Remark 1 If (17) is disconjugate on $\left[T_{1}, \infty\right), T_{1} \geq T$, then any principal solution of (17) does not have zeros on $\left(T_{1}, \infty\right)$, see [21, Chapter XI,Exercise 6.6]. Thus, a necessary condition for positiveness of the principal solution on the open interval $(T, \infty)$ is the disconjugacy of the equation. Nevertheless, disconjugacy cannot be sufficient for the positiveness of principal solution on the close half-line $[T, \infty)$, as the following example shows.

Example 1 Consider the equation

$$
\left(a(t) y^{\prime}\right)^{\prime}+y=0, \quad t \geq 0,
$$

where $a(1)=1$ and

$$
a(t)=\frac{1+t-2 e^{t-1}}{1-t} \text { if } t \neq 1 .
$$

Hence, $a$ is a positive continuous function on $[0, \infty)$ and (3) holds for $a$. Using (19), we get that $y_{0}(t)=t e^{-t}$ is the principal solution of (20). Moreover, in view of Lemma 3, equation (20) is disconjugate on $[0, \infty)$.

Consider now the special case $\beta(t) \equiv M>0$ in (17), i.e., the equation

$$
\left(a(t) y^{\prime}\right)^{\prime}+M y=0 .
$$

In view of Example 1, the disconjugacy of (L) on $[T, \infty)$ does not guarantee the positiveness of principal solution at the initial point $t=T$. To obtain this additional property, consider the so-called dual equation to $(\mathrm{L})$, that is the equation

$$
v^{\prime \prime}+\frac{M}{a(t)} v=0
$$

which is obtained from (L) by the change of variable $v(t)=a(t) y^{\prime}(t)$. The dual equation has been often used in the literature for studying oscillatory properties of second-order selfadjoint linear equations, see, e.g., [8,9,26], and, for the half-linear case, [10,11,18].

The following necessary and sufficient condition for the disconjugacy of (D) holds, see also [21, page 352].

Lemma 4 Equation $(D)$ is disconjugate on $[T, \infty)$ if and only if $(D)$ has a solution $v_{0}$ such that $v_{0}(t)>0$ on $(T, \infty)$ and $v_{0}^{\prime}(t)>0$ on $[T, \infty)$. 
Proof Assume that (D) is disconjugate on $[T, \infty)$. From Lemma 3, there exists a solution $v_{0}$ of (D) such that $v_{0}(t)>0$ for $t>T$. Thus, $v_{0}^{\prime}$ is decreasing for $t>T$. We claim that $v_{0}^{\prime}(t)>0$ on the whole interval $[T, \infty)$. By contradiction, if $v_{0}^{\prime}$ has a zero on $[T, \infty)$, then there exists $t_{1}>T$ such that $v_{0}^{\prime}(t) \leq v_{0}^{\prime}\left(t_{1}\right)<0$ for $t \geq t_{1}$. Integrating this inequality, we get $v_{0}(t) \leq v_{0}\left(t_{1}\right)+v_{0}^{\prime}\left(t_{1}\right)\left(t-t_{1}\right)$, which gives a contradiction with the positiveness of $v_{0}$ when $t$ tends to infinity. The opposite statement follows again in virtue of Lemma 3.

From Lemma 4 we obtain the following.

Lemma 5 If $(D)$ is disconjugate on $[T, \infty)$, then $(L)$ has a principal solution $y_{0}$ such that $y_{0}(t)>0$ on $[T, \infty)$ and $y_{0}^{\prime}(t)<0$ on $(T, \infty)$.

Proof In view of Lemma 4 and the change of variable $y(t)=v^{\prime}(t)$, equation (L) has a solution $y_{0}$ which satisfies $y_{0}(t)>0$ on $[T, \infty)$ and $y_{0}^{\prime}(t)<0$ on $(T, \infty)$. Hence, the disconjugacy of (L) follows from Lemma 3. If $y_{0}$ is not principal solution, from [21, Corollary 6.3] the solution $\bar{y}$ given by

$$
\bar{y}(t)=y_{0}(t) \int_{t}^{\infty} \frac{\mathrm{d} s}{a(s) y_{0}^{2}(s)},
$$

is the desired principal solution of $(\mathrm{L})$.

Remark 2 Example 1 shows that the assumption on disconjugacy of (D) in Lemma 5 cannot by replaced by the disconjugacy of (L). Moreover, observe that the dual equation of (20) is

$$
v^{\prime \prime}+a^{-1}(t) v=0,
$$

where $a$ is defined in Example 1. It is easy to verify that the function $v_{0}(t)=2 e^{-1}-(1+t) e^{-t}$ is a principal solution of (21). Since $v_{0}(1)=0$, any principal solution of (21) has a zero at $t=1$. Consequently, (21) is not disconjugate on $[0, \infty)$.

\section{An auxiliary BVP on $[1, \infty)$}

For any $c>0$, consider for $t \geq 1$ the existence of solutions $x$ of (1) which satisfy the boundary conditions

$$
x(1)=c, x^{\prime}(1) \leq 0, \quad x(t)>0 \text { on }[1, \infty), \quad \lim _{t \rightarrow \infty} x(t)=0 .
$$

The solvability of this BVP is based on a general fixed point theorem for operators defined in a Fréchet space, see [12, Theorem 1.3]. In particular, this result reduces the existence of solutions of a BVP for differential equations on noncompact intervals to the existence of suitable a-priori bounds and it is mainly useful when the associated fixed point operator is not known in an explicit form. We recall this result in the form that will be used.

Theorem 2 Consider the BVP on $[T, \infty), T \geq 0$,

$$
\left(a(t) x^{\prime}\right)^{\prime}+b(t) F(x)=0, \quad x \in S,
$$

where $S$ is a nonempty subset of the Fréchet space $C[T, \infty)$. Let $G$ be a continuous function on $\mathbb{R}^{2}$, such that $F(d)=G(d, d)$ for any $d \in \mathbb{R}$ and assume that there exists a nonempty, closed, convex and bounded subset $\Omega \subset C[T, \infty)$ such that for any $u \in \Omega$ the BVP on $[T, \infty)$

$$
\left(a(t) x^{\prime}\right)^{\prime}+b(t) G(u(t), x(t))=0, \quad x \in S
$$


admits a unique solution $x_{u}$. Let $\Psi$ be the operator $\Omega \rightarrow C[T, \infty)$, such that $\Psi(u)=x_{u}$. Assume

$\left(i_{1}\right) \Psi(\Omega) \subset \Omega$;

$\left(i_{2}\right)$ if $\left\{u_{n}\right\} \subset \Omega$ is a sequence converging in $\Omega$ and $\Psi\left(u_{n}\right) \rightarrow x$, then $x \in S$.

Then $\Psi$ has a fixed point in $\Omega$, which is a solution of the $B V P(23)$.

Let $\widetilde{F}$ be the function

$$
\widetilde{F}(v)=\frac{F(v)}{v} \text { if } v>0, \quad \widetilde{F}(0)=k_{0},
$$

where $k_{0}$ is defined in (6) and set $b_{+}(t)=\max \{b(t), 0\}, b_{-}(t)=-\min \{b(t), 0\}$. Thus $b(t)=b_{+}(t)-b_{-}(t)$. The following holds.

Theorem 3 Assume that Eq. (8) is disconjugate on $[1, \infty)$. Then, for any $c>0, E q$. (1) has a unique globally positive decreasing solution $x$ on $[1, \infty)$ satisfying $(22)$ and (11).

Proof Fixed $c>0$, consider the equations

$$
\begin{aligned}
\left(a(t) y^{\prime}\right)^{\prime}+B y & =0, \\
\left(a(t) w^{\prime}\right)^{\prime}-b_{-}(t) w & =0 .
\end{aligned}
$$

From Lemma 5, Eq. (25) is disconjugate on $[1, \infty)$ and has a principal solution $y_{0}$ such that $y_{0}(1)=c, y_{0}(t)>0$ on $[1, \infty), y_{0}^{\prime}(t)<0$ on $(1, \infty)$. Moreover, from [9, Theorem 1] we obtain $\lim _{t \rightarrow \infty} y_{0}(t)=0$.

Since $-b_{-}(t) \leq 0$, Eq. (25) is a Sturm majorant for (26). Thus (26) has a positive principal solution $w_{0}$ such that $w_{0}(1)=c, w_{0}^{\prime}(t) \leq 0$ for $t \geq 1$, see, e.g., [21, Corollary 6.4]. Using the comparison result for the principal solutions, see e.g., [21, Corollary 6.5], we get on $(1, \infty)$

$$
\frac{w_{0}^{\prime}(t)}{w_{0}(t)} \leq \frac{y_{0}^{\prime}(t)}{y_{0}(t)}
$$

and so $0<w_{0}(t) \leq y_{0}(t)$ for $t \geq 1$.

Let $\Omega$ and $S$ be the subsets of the Fréchet space $C[1, \infty)$ given by

$$
\begin{aligned}
& \Omega=\left\{u \in C[1, \infty), \frac{1}{2} w_{0}(t) \leq u(t) \leq y_{0}(t)\right\}, \\
& S=\left\{x \in C[1, \infty), x(1)=c, \quad x(t)>0, \quad \int_{1}^{\infty} \frac{1}{a(t) x^{2}(t)} d t=\infty\right\},
\end{aligned}
$$

respectively.

For any $u \in \Omega$, consider the linear equation

$$
\left(a(t) x^{\prime}\right)^{\prime}+b(t) \widetilde{F}(u(t)) x(t)=0,
$$

where $\widetilde{F}$ is given in (24). In view of (5), we have $\sup _{v \geq 0} \widetilde{F}(v) \leq 1$. Hence, (25) is a majorant for (27). Thus, using again the comparison result [21, Corollary 6.5], Eq. (27) has a unique positive principal solution $x_{u}$, such that $x_{u}(1)=c$, and for $t>1$

$$
\frac{x_{u}^{\prime}(t)}{x_{u}(t)} \leq \frac{y_{0}^{\prime}(t)}{y_{0}(t)} .
$$

Hence, taking into account that $y_{0}$ is decreasing to zero as $t$ tends to infinity, we get

$$
\begin{aligned}
& 0<x_{u}(t) \leq y_{0}(t) \quad \text { on }[1, \infty) \\
& \lim _{t \rightarrow \infty} x_{u}(t)=0, x_{u}^{\prime}(t)<0 \text { on }(1, \infty)
\end{aligned}
$$


Thus, for any $u \in \Omega$, Eq. (27) has a solution $x_{u} \in S$, which is unique in view of (19).

Denote by $\Psi: \Omega \rightarrow C[1, \infty)$ the operator

$$
\Psi(u)=x_{u} .
$$

Using again the comparison result [21, Corollary 6.5] for Eqs. (27) and (26), we obtain for any $u \in \Omega$ and $t \geq 1$

$$
\frac{w_{0}^{\prime}(t)}{w_{0}(t)} \leq \frac{x_{u}^{\prime}(t)}{x_{u}(t)}
$$

Then, in view of (28) we get for any $u \in \Omega$ and $t \geq 1$

$$
w_{0}(t) \leq x_{u}(t) \leq y_{0}(t),
$$

i.e., the operator $\Psi$ maps $\Omega$ into itself.

Now, let $\left\{u_{n}\right\} \subset \Omega$ be a sequence converging in $\Omega$ and $x_{u_{n}}=\Psi\left(u_{n}\right) \rightarrow x$. Clearly $x(1)=c$. Since $\overline{\Psi(\Omega)} \subset \Omega$, we get $x(t)>0$. Moreover, since $y_{0}$ is a principal solution of (25), from (28) we obtain

$$
\int_{1}^{\infty} \frac{1}{a(t) x^{2}(t)} d t \geq \int_{1}^{\infty} \frac{1}{a(t) y_{0}^{2}(t)} d t=\infty .
$$

Thus, $x \in S$ and, by Theorem 2, there exists a fixed point $\bar{x}$ of $\Psi$ in $\Omega$. Clearly, $\bar{x}$ is a solution of (1) on $[1, \infty)$ and $\bar{x}(1)=c$. Since $\bar{x}$ is also a principal solution of (27) with $u=\bar{x}$, from (28) we get $\bar{x}(t)>0, \bar{x}^{\prime}(t)<0$ for $t>1, \bar{x}^{\prime}(1) \leq 0$ and $\lim _{t \rightarrow \infty} \bar{x}(t)=0$. Thus $\bar{x}$ is positive decreasing on $(1, \infty)$ and satisfies $(22)$ and $(11)$.

Finally, it remains to verify that (1) has a unique solution which satisfies (22). Let $x, v$ be two positive solutions of (1) defined on $[1, \infty)$ and satisfying (22). In view of the first part of the proof, we can suppose also that

$$
\int_{1}^{\infty} \frac{\mathrm{d} t}{a(t) x^{2}(t)}=\infty
$$

Denote by $\Phi(u, v)$ the function $(u \geq 0, v \geq 0)$

$$
\Phi(u, v)= \begin{cases}(F(u)-F(v)) /(u-v) & \text { if } u \neq v \\ \mathrm{~d} F(u) / \mathrm{d} u & \text { if } u=v\end{cases}
$$

and set $z(t)=x(t)-v(t)$. Thus, $z$ is a solution of the equation

$$
\left(a(t) z^{\prime}\right)^{\prime}+b(t) \bar{\Phi}(t) z=0,
$$

where $\bar{\Phi}(t)=\Phi(x(t), v(t))$. In virtue of (5), we have

$$
b(t) \bar{\Phi}(t) \leq B .
$$

Since, from Lemma 5, Eq. (25) is disconjugate on $[1, \infty)$, Eq. (31) is disconjugate on $[1, \infty)$ too. Since $z(1)=0$, the solution $z$ does not have zeros for $t>1$ and so, without loss of generality, we can suppose $z(t)>0$ for $t>1$. Because $\lim _{t \rightarrow \infty} z(t)=0$, there exists $t_{1}>1$ such that $z^{\prime}\left(t_{1}\right)=0$. Moreover, taking into account that $x$ satisfies (30) and $z(t)<x(t)$, we get that $z$ is a principal solution of (31). Using again the comparison result [21, Corollary 6.5] for Eqs. (25) and (31), we obtain for $t>1$

$$
\frac{z^{\prime}(t)}{z(t)} \leq \frac{y_{0}^{\prime}(t)}{y_{0}(t)}
$$


where $y_{0}$ is the positive decreasing principal solution of (25) defined in the first part of the proof. Thus, the inequality (32) gives a contradiction at $t=t_{1}$, because

$$
\frac{y_{0}^{\prime}\left(t_{1}\right)}{y_{0}\left(t_{1}\right)}<0 .
$$

We conclude this section with the following continuity result for starting points of solutions of (1) which satisfy (22).

Theorem 4 Assume that Eq. (8) is disconjugate on $[1, \infty)$. Let $\left\{c_{n}\right\}$ be a positive sequence converging to zero and denote by $x_{n}$ the unique solution of (1) which satisfies (22) with $c_{n}=c$. Then the sequence $\left\{x_{n}^{\prime}(1)\right\}$ converges to zero.

Proof In virtue of Theorem 3, for any $c_{n}>0$, Eq. (1) has a unique solution $x_{n}$ which satisfies (22) with $c_{n}=c$. Denote by $w_{n}$ the principal solution of (26) such that $w_{n}(1)=c_{n}$. From (29) and (22), we get

$$
w_{n}^{\prime}(1) \leq x_{n}^{\prime}(1) \leq 0 \text {. }
$$

Since principal solutions are determined up to a constant factor, we have

$$
w_{n}(t)=\frac{c_{n}}{c_{1}} w_{1}(t)
$$

Hence, $w_{n}^{\prime}(1)=c_{n} w_{1}^{\prime}(1) / c_{1}$ and from (33) the assertion follows

\section{Proof of the main result}

In this section, we prove Theorem 1 and we show some of its consequences. To this aim, the following generalization of the well known Kneser's theorem (see for instance [13, Section 1.3]), plays a key role.

Proposition 2 Consider the system

$$
z^{\prime}=F(t, z), \quad(t, z) \in\left[T_{1}, T_{2}\right] \times \mathbb{R}^{n}
$$

where $F$ is continuous and bounded, and let $K_{0}$ be a continuum (i.e., a compact and connected subset) of $\left\{\left(T_{1}, w\right): w \in \mathbb{R}^{n}\right\}$. Let $\mathcal{Z}\left(K_{0}\right)$ be the family of all the solutions emanating from $K_{0}$. If any solution $z \in \mathcal{Z}\left(K_{0}\right)$ is defined on the whole interval $\left[T_{1}, T_{2}\right]$, then the cross-section $\mathcal{Z}\left(T_{2} ; K_{0}\right)=\left\{z\left(T_{2}\right): z \in \mathcal{Z}\left(K_{0}\right)\right\}$ is a continuum in $\mathbb{R}^{n}$.

Proof of Theorem 1 Let $x_{1} \in \Delta_{1}$ and $x_{2} \in \Delta_{2}$ be the solutions on $[0,1]$ of (1), whose existence is guaranteed by Proposition 1 , and let $\alpha=\max \left\{x_{1}^{\prime}(0), x_{2}^{\prime}(0)\right\}>0, \beta=$ $\min \left\{x_{1}^{\prime}(0), x_{2}^{\prime}(0)\right\}>0$. Put

$$
L=\alpha a(0) A(1)
$$

and let $\widehat{F}$ be a Lipschitz function on $\mathbb{R}$ such that

$$
\widehat{F}(u)= \begin{cases}0, & u<0 \\ F(u), & 0 \leq u \leq L . \\ F(L), & u>L\end{cases}
$$


For $\ell \in(0, \alpha]$, consider the Cauchy problem

$$
\left\{\begin{array}{l}
\left(a(t) x^{\prime}\right)^{\prime}+b(t) \widehat{F}(x)=0, \quad t \in[0,1] \\
x(0)=0, x^{\prime}(0)=\ell,
\end{array}\right.
$$

and denote by $x_{\ell}$ the unique solution of (35). Let us show that $x_{\ell}$ is defined on the whole interval $[0,1]$. For any solution $x$ of the equation in $(35)$, the function $a(\cdot) x^{\prime}(\cdot)$ is nonincreasing, so $a(t) x_{\ell}^{\prime}(t) \leq a(0) x_{\ell}^{\prime}(0)=a(0) \ell$. Integrating this inequality, in view of (34) we get for $t \in[0,1]$

$$
x_{\ell}(t) \leq a(0) \quad \ell \int_{0}^{t} \frac{1}{a(s)} \mathrm{d} s \leq a(0) \ell A(1) \leq L .
$$

Assume now that $x_{\ell}(t)>0$ on $\left(0, t_{1}\right), 0<t_{1} \leq 1$, and $x_{\ell}\left(t_{1}\right)=0$. Then, in virtue of the uniqueness of the Cauchy problem (35), we obtain $x_{\ell}^{\prime}\left(t_{1}\right)<0$. If $t_{1}<1$, then $x_{\ell}(t)<0$ in a right neighborhood of $t_{1}$ and satisfies $\left(a(t) x_{\ell}^{\prime}\right)^{\prime}=0$, which gives $x_{\ell}(t)<0$ for every $t \geq t_{1}$ for which this solution exists. Since $x_{\ell}^{\prime}\left(t_{1}\right)<0$, by integration we obtain for $t>t_{1}$

$$
x_{\ell}(t)=a\left(t_{1}\right) x_{\ell}^{\prime}\left(t_{1}\right) \int_{t_{1}}^{t} \frac{1}{a(s)} \mathrm{d} s>a\left(t_{1}\right) x_{\ell}^{\prime}\left(t_{1}\right) A(1),
$$

that is, $x_{\ell}$ is bounded from below. Therefore, the solution $x_{\ell}$ of (35) is defined on the whole interval $[0,1]$.

Let $x$ be any solution of (1), nonnegative on $[0,1]$ and satisfying $x(0)=0, x^{\prime}(0)=\ell \in$ $(0, \alpha]$. Then $x$ is also a solution of (35) for $0 \leq t \leq 1$, and vice versa. Indeed, reasoning as above, we obtain $x(t) \leq L$ on $[0,1]$ and therefore $F(x(t))=\widehat{F}(x(t))$ for all $t \in[0,1]$.

Put $K_{0}=\left\{\left(x(1), x^{\prime}(1)\right): x\right.$ is solution of (35) with $\left.\ell \in[\beta, \alpha]\right\}$. Since any solution of (35) is defined on the whole $[0,1]$, by Proposition 2 the set $K_{0}$ is a continuum in $\mathbb{R}^{2}$, containing the points $\left(0, x_{1}^{\prime}(1)\right),\left(x_{2}(1), 0\right)$, with $x_{1}^{\prime}(1)<0, x_{2}(1)>0$. Further, $K_{0}$ does not contain any point $(0, c)$ with $c \geq 0$. Therefore, a continuum $K_{1} \subseteq K_{0}$ exists, $K_{1} \subseteq \bar{\pi}=\{(u, v): u \geq$ $0, v \leq 0\},(0,0) \notin K_{1}$, and there exist two points $P, Q \in K_{1}, P=(p, 0), Q=(0,-q)$, $p>0, q>0$.

In order to complete the proof, we use a similar argument to the one given in [24, Theorem 1.1], with minor changes. Consider Eq. (1) for $t \geq 1$. By Theorem 3, for every $c>0$, (1) has a unique positive decreasing solution $x$ satisfying (22) and (11). Then, the set $S_{1}$ of the initial data of the solutions of (1) on $[1, \infty)$ satisfying (11) and (22) is connected, $S_{1} \subset \bar{\pi}$, and its projection on the first component is the half-line $(0, \infty)$. Further, from Theorem 4 , $(0,0) \in \bar{S}_{1}$. Therefore, we have

$$
K_{1} \cap S_{1} \neq \varnothing .
$$

Let us show that each point $(c, d) \in K_{1} \cap S_{1}$ corresponds to a solution of the BVP (1)-(2). Let $(c, d) \in K_{1} \cap S_{1}$. Then $c>0, d \leq 0$. Since $(c, d) \in K_{1}$, there exists a solution $u$ of (35), for a suitable $\ell \in[\beta, \alpha]$, such that $u(1)=c>0$ and $u^{\prime}(1)=d \leq 0$. Since $u(1)>0$ we have $u(t)>0$ on $(0,1]$. Therefore, $u$ is also a solution of $(1)$ in $[0,1]$, with $u(0)=0, u(t)>0$ for $t \in(0,1]$. As $(c, d) \in S_{1}$, a positive decreasing solution $v$ of (1) exists on $[1, \infty)$, which satisfies (22) and $v(1)=c=u(1), v^{\prime}(1)=d=v^{\prime}(1)$. Hence, the function

$$
x(t)= \begin{cases}u(t), & t \in[0,1], \\ v(t), & t>1 .\end{cases}
$$

is a solution of the BVP (1)-(2) and the proof is complete. 
From Theorem 1 and Proposition 1, we get the following.

Corollary 1 Let assumptions of Proposition 1-(ii) be satisfied and Eq. (8) be disconjugate on $[1, \infty)$. Then the BVP (1)-(2) has a solution.

We close this section with the solvability of our BVP for the perturbed equation

$$
\left(a(t) z^{\prime}\right)^{\prime}+\left(b(t)+b_{1}(t)\right) F(z)=0,
$$

where $b_{1}$ is a continuous function for $t \geq 0$ such that $b_{1}(t) \equiv 0$ on $[0,1]$ and $b_{1}(t) \leq 0$ for $t>1$.

Corollary 2 If assumptions of Theorem 1 are satisfied, then Eq. (36) has a solution z satisfying boundary conditions (2).

\section{Examples and concluding remarks}

Theorem 1 is illustrated by the following example.

Example 2 Consider the equation

$$
\left(a(t) x^{\prime}\right)^{\prime}+b(t) F(x)=0,
$$

where

$$
a(t)=(1+t)^{2}, \quad b(t)=\frac{1}{5 e} \exp \left(\frac{16}{1+16 t^{4}}\right) \cos \left(\frac{\pi t}{2}\right) \quad \text { for } t \geq 0 .
$$

and $F$ satisfies (5) and (6) with

$$
k_{0}=\frac{9}{e^{15}}, \quad k_{\infty}=1 .
$$

Since $b$ is decreasing on $[0,1]$, we get

$$
\int_{1 / 3}^{1 / 2} b(\tau) \mathrm{d} \tau \geq \frac{1}{6} b(1 / 2)=\frac{\sqrt{2}}{60} e^{7} .
$$

For Eq. (37), the function $A$ in (7) becomes

$$
A(t)=\frac{t}{1+t},
$$

so assumptions (9), (10) are verified for $t_{1}=1 / 3$ and $t_{2}=1 / 2$, because

$$
\begin{aligned}
& A(1)|b|_{L} \geq \frac{1}{2} b(0)=\frac{e^{15}}{10}, \\
& \frac{A(1)}{A\left(t_{1}\right)\left(A(1)-A\left(t_{2}\right)\right)}=12<\frac{\sqrt{2}}{60} e^{7} .
\end{aligned}
$$

Finally, for $t \in[1, \infty)$ we have

$$
b(t) \leq \frac{1}{5 e}<\frac{1}{4}
$$

and Eq. (8) becomes the Euler equation

$$
v^{\prime \prime}+\frac{1}{4(1+t)^{2}} v=0
$$


which is disconjugate on $[1, \infty)$, see, e.g., [26, Chapter 2.1]. Hence, in view of Theorem 1 , Eq. (37) has solutions $x$ which satisfy the boundary conditions (2) and

$$
\int_{1}^{\infty} \frac{1}{(1+t)^{2} x^{2}(t)} \mathrm{d} t=\infty
$$

Remark 3 Example 2 can be slightly modified for the nonlinearity

$$
F(u)=\frac{u^{2}}{1+u}
$$

or the nonlinearity

$$
F(u)=\frac{u}{1+\sqrt{u}} .
$$

Remark 4 Consider the equation

$$
\left(a(t) x^{\prime}\right)^{\prime}+\left(b(t)+b_{1}(t)\right) F(x)=0,
$$

where the functions $a, b$ are given in (38), $b_{1}$ is the function

$$
b_{1}(t)=\left(e-e^{t}\right)(|\cos t|-\cos t) \text { for } t \geq 1, \quad b_{1}(t) \equiv 0 \text { for } t \in[0,1),
$$

and $F$ is as in Example 2. Since $b_{1}(t) \leq 0$, in view of Corollary 2, Eq. (39) has solutions $x$ which satisfy the boundary conditions (2).

Remark 5 Theorem 1 and Corollaries 1, 2 continue to hold if the assumption (5) is replaced by the more general condition

$$
\exists K>0: 0 \leq \frac{\mathrm{d} F(u)}{\mathrm{d} u} \leq K \text { for } u \geq 0
$$

and the disconjugacy of $(8)$ is substituted by the disconjugacy on $[1, \infty)$ of the linear equation

$$
v^{\prime \prime}+\frac{B K}{a(t)} v=0 .
$$

Remark 6 The assumption $k_{0} \neq k_{\infty}$ implies that $F$ cannot be a linear function on $[0, \infty)$. If the linear equation

$$
\left(a(t) x^{\prime}\right)^{\prime}+b(t) x=0
$$

has a solution $x$ satisfying (2), then in virtue of Lemma 3, (40) is disconjugate on $[0, \infty)$. However, $x$ is not necessarily the principal solution of (40). The following example illustrates this case.

Example 3 Consider the equation

$$
\left(e^{2 t} x^{\prime}\right)^{\prime}+e^{2 t} x=0, \quad t \geq 0
$$

A standard calculation shows that $x_{0}(t)=e^{-t}, x_{1}(t)=t e^{-t}$ are solutions of (41). Obviously, $x_{1}$ satisfies (2). Observe that $x_{1}$ is a nonprincipal solution and $x_{0}$ is the principal solution.

In a forthcoming paper, we will consider this kind of BVPs for nonlinear equations for which $k_{0}=k_{\infty}$.

Acknowledgements Research of the first author was supported by the Grant GAP 201/11/0768 of the Czech Grant Agency. Research of the third author was supported by the Gruppo Nazionale per l'Analisi Matematica, la Probabilità e le loro Applicazioni (GNAMPA) of the Italian Istituto Nazionale di Alta Matematica (INdAM). 


\section{References}

1. Agarwal, R.P., O’Regan, D.: Infinite Interval Problems for Differential, Difference and Integral Equations. Kluwer, Dordrecht (2001)

2. Andres, J., Gabor, G., Górniewicz, L.: Boundary value problems on infinite intervals. Trans. Am. Math. Soc. 351, 4861-4903 (1999)

3. Bartolo, R., Candela, A.M., Salvatore, A.: Perturbed asymptotically linear problems. Ann. Mat. Pura Appl. (4) 193, 89-101 (2014)

4. Boscaggin, A., Zanolin, F.: Pairs of positive periodic solutions of second order nonlinear equations with indefinite weight. J. Differ. Equ. 252, 2900-2921 (2012)

5. Boscaggin, A., Zanolin, F.: Second-order ordinary differential equations with indefinite weight: the Neumann boundary value problem. Ann. Mat. Pura Appl. (2013). doi:10.1007/s10231-013-0384-0

6. Calamai, A., Infante, G.: Nontrivial solutions of boundary value problems for second-order functional differential equations. Ann. Mat. Pura Appl. (2013). doi:10.1007/s10231-015-0487-x

7. Cortázar, C., Dolbeault, J., García-Huidobro, M., Manásevich, R.: Existence of sign changing solutions for an equation with a weighted p-Laplace operator. Nonlinear Anal. 110, 1-22 (2014)

8. Cecchi, M., Marini, M., Villari, G.: On monotonicity property for a certain class of second order differential equations. J. Differ. Equ. 82, 15-27 (1989)

9. Cecchi, M., Marini, M., Villari, G.: Integral criteria for a classification of solutions of linear differential equations. J. Differ. Equ. 99, 381-397 (1992)

10. Cecchi, M., Došlá, Z., Marini, M.: Half-linear equations and characteristic properties of the principal solution. J. Differ. Equ. 208, 494-507 (2005)

11. Cecchi, M., Došlá, Z., Marini, M.: Corrigendum to: "Half-linear equations and characteristic properties of the principal solutions" [J. Differential Equations 208 (2005), 494-507]. J. Differ. Equ. 221, 272-274 (2006)

12. Cecchi, M., Furi, M., Marini, M.: On continuity and compactness of some nonlinear operators associated with differential equations in noncompact intervals. Nonlinear Anal. Theory Methods Appl. 9, 171-180 (1985)

13. Coppel, W.A.: Stability and Asymptotic Behavior of Differential Equations. D. C. Heath and Co., Boston (1965)

14. Coppel, W.A.: Disconjugacy, Lecture Notes Mathematics, vol. 220. Springer, Berlin (1971)

15. Došlá, Z., Marini, M., Matucci, S.: On some boundary value problems for second order nonlinear differential equations. Math. Bohem. 137, 113-122 (2012)

16. Došlá, Z., Marini, M., Matucci, S.: A boundary value problem on a half-line for differential equations with indefinite weight. Commun. Appl. Anal. 15, 341-352 (2011)

17. Došlá, Z., Marini, M., Matucci, S.: Positive solutions of nonlocal continuous second order BVP's. Dyn. Syst. Appl. 23, 431-446 (2014)

18. Došlý, O., Řehák, P.: Half-linear Differential Equations, North-Holland, Mathematics Studies, vol. 202. Elsevier Sci. B.V, Amsterdam (2005)

19. Erbe, L.H., Wang, H.: On the existence of positive solutions of ordinary differential equations. Proc. Am. Math. Soc. 120, 743-748 (1994)

20. Gaudenzi, M., Habets, P., Zanolin, F.: An example of a superlinear problem with multiple positive solutions. Atti Sem. Mat. Fis. Univ. Modena 51, 259-272 (2003)

21. Hartman, P.: Ordinary Differential Equations, 2nd edn. Birkäuser, Boston-Basel-Stuttgart (1982)

22. Kiguradze, I.T., Chanturia, A.: Asymptotic Properties of Solutions of Nonautonomous Ordinary Differential Equations. Kluwer Acad. Publ. G, Dordrecht (1993)

23. Lan, K., Webb, J.R.L.: Positive solutions of semilinear differential equations with singularities. J. Differ. Equ. 148, 407-421 (1998)

24. Marini, M., Matucci, S.: A boundary value problem on the half-line for superlinear differential equations with changing sign weight. Rend. Istit. Mat. Univ. Trieste 44, 117-132 (2012)

25. Matucci S.: A new approach for solving nonlinear BVP's on the half-line for second order equations and applications. Math. Bohem. 140, 153-169 (2015)

26. Swanson, C.A.: Comparison and Oscillation Theory of Linear Differential Equations. Academic Press, New York (1968)

27. Wei, Y., Wong, P.J.Y.: Existence and uniqueness of solutions for delay boundary value problems with p-Laplacian on infinite intervals. Bound. Value Probl. 2013, 141 (2013) 